\title{
Structure and antigenicity of lipoarabinomannan from Mycobacterium bovis BCG
}

\author{
Susi Prinzis, $\uparrow$ Delphi Chatterjee and Patrick J. Brennan* \\ Department of Microbiology, Colorado State University, Fort Collins, Colorado 80523, USA
}

(Received 18 May 1993; accepted 1 June 1993)

\begin{abstract}
Lipoarabinomannan (LAM), a major lipoglycan of the mycobacterial cell envelope, was previously recognized as existing in two major forms: LAM with arabinofuranosyl (Araf)-containing termini (AraLAM) and a mannosecapped version (ManLAM) in which the majority of these termini are modified by additional mannose residues. Since ManLAM was first recognized in the virulent (Erdman) strain of Mycobacterium tuberculosis and the noncapped version in a rapidly growing, attenuated, H37Ra strain, it was thought that mannose capping may be a key factor in virulence. In the present study, LAM from $M$. bovis BCG was isolated and the non-reducing termini sequenced through differential $O$-alkylation, partial depolymerization and gas chromatography-mass spectrometric analyses of fragments. LAM from $M$. bovis BCG contains a short mannan backbone, highly branched arabinofuranosyl-containing side chains and several mannosyl residues capping the non-reducing termini of these side chains. Thus, LAM from M. bovis BCG is of the ManLAM type, showing no major structural differences at the non-reducing ends from the $M$. tuberculosis Erdman product. This observation led us to examine the earlier strain and to conclude that it showed little resemblance to conventional strains of $M$. tuberculosis. Thus, the absence of mannose caps may be more a feature of rapid growth than of avirulence. These results demonstrate that the relationship between mannose capping and disease induction is not a simple one. However, use of a panel of LAM-specific monoclonal antibodies showed antigenic differences between the BCG and the Erdman products, suggesting the presence of features specific to the different strains and pointing to LAM as a molecule within which further species and strain variations reside.
\end{abstract}

\section{Introduction}

Among the carbohydrates of the mycobacterial cell wall, two polymers show strong antigenicity: arabinomannan and lipoarabinomannan (LAM) (Hunter et al., 1986). LAM is widely distributed among Mycobacterium spp. (Misaki et al., 1977), and its antigenic determinants are shared extensively among individual species (Gaylord $e t$ al., 1987). Several investigations have focused on its wide biological activities. LAM from both Mycobacterium tuberculosis and Mycobacteria leprae is known to suppress T-cell proliferation (Kaplan et al., 1987;

*Author for correspondence. Tel. +1 3034916700 ; fax, +1303491 1815.

†Present address: Laboratoire de Biochimie Microbienne, Université Claud Bernard, Lyon, France.

Abbreviations: LAM, lipoarabinomannan; AraLAM, LAM with arabinofuranosyl-containing termini; ManLAM, mannose-capped LAM (see text); dLAM, deacylated LAM; TNF, tumour necrosis factor.
Moreno et al., 1988; Molloy et al., 1990), to inhibit $\gamma$ interferon-mediated activation of macrophages (Sibley $e t$ al., 1988, 1990), to inhibit the synthesis of mRNA encoding IL-2, IL-5 and GM-CSF in human T cells (Chujor et al., 1992), and to enhance the production of tumour necrosis factor (TNF) by mononuclear cells (Moreno et al., 1989; Barnes et al., 1990, 1992; Chatterjee et al., 1992c). These and other results combined suggest that LAM may have broad immunosuppressive effects in mycobacterioses and contribute to the disease state (Levis et al., 1987; Moreno et al., 1988, 1989; Chan et al., 1991).

Recently, LAM from what was believed to be a strain of M. tuberculosis H37Ra (Chatterjee et al., 1991) and M. tuberculosis Erdman (Chatterjee et al., 1992b) were described. Both products contain a phosphatidylinositol unit at the reducing end (Chatterjee et al., 1992a), a mannan backbone with 6- and 2,6-linked mannopyranoses, and several arabinofuranosyl-containing chains marked by extensive branching. The non-reducing termini of the arabinan portion of LAM from the earlier $\mathrm{H} 37 \mathrm{Ra}$ strain consist of either of two arrangements, $\beta$-D- 
Araf-( $1 \rightarrow 2)-\alpha$-D-Araf- $(1 \rightarrow 5)-\alpha$-D-Ara $f \rightarrow$ or $[\beta$-D-Ara $f-$ $(1 \rightarrow 2)-\alpha$-D-Araf-( $(1 \rightarrow]_{2}-(3$ and 5$)-\alpha$-D-Ara $\rightarrow \rightarrow$; this product can be named AraLAM. In another strain (Erdman) of $M$. tuberculosis, the arabinan ends of LAM are extensively capped with single or di-, tri- or tetramannosyl units (Chatterjee et al., 1992b). Consequently, we had termed this form of LAM as mannose-capped LAM or ManLAM and had speculated that these mannose residues may be implicated in the successful colonization or disease induction of strains of $M$. tuberculosis, a view reinforced by the observation that ManLAM is the dominant form present in $M$. avium (unpublished data) and, apparently, in $M$. paratuberculosis (Sugden et al., 1987). It was thus of interest to investigate the structure of LAM from a vaccine strain of the $M$. tuberculosis $-M$. bovis complex whose LAM might a priori be expected to be of the type associated with nonvirulence.

\section{Methods}

Isolation and purification of LAM from M. bovis BCG (Eli Lilly strain). The supernatant obtained after breakage of cells and centrifugation at $27000 \mathrm{~g}$ was precipitated with $90 \%(\mathrm{v} / \mathrm{v})$ acetone. The acetone precipitate was applied to a column $(1.5 \times 100 \mathrm{~cm})$ of Sephacryl S-400 (Bio-Rad). Fractions enriched in carbohydrates were eluted with $6 \mathrm{M}$-guanidine. $\mathrm{HCl}$ in $10 \mathrm{~mm}$-Tris/ $\mathrm{HCl}, \mathrm{pH} \mathrm{7.4}$, dialysed, concentrated on an Amicon flowcell (Amicon 8200), and applied to a column $(1.5 \times 100 \mathrm{~cm})$ of Sephacryl S-200 (Bio-Rad). Separation of LAM from lipomannan and phosphatidylinositolmannosides was achieved by elution with $10 \mathrm{~mm}$-Tris $/ 0.2 \mathrm{M}-\mathrm{NaCl}$ buffer containing $10 \mathrm{~mm}$-EDTA, $0.02 \% \mathrm{NaN}_{3}$ and $0.25 \%$ deoxycholate. Fractions were monitored by SDS-PAGE. LAM-containing fractions were pooled, dialysed, concentrated and dried. In order to remove protein contaminants, phenol extraction was performed (Hunter et al., 1986). Purified LAM thus obtained was subjected to chemical and immunochemical analyses.

The homogeneity of LAM from BCG was tested by HPLC. Gel filtration chromatography was conducted on a column $(250 \times 4.6 \mathrm{~mm})$ of SynchroPack GPC 300 (Synchrom), with 0.1 M-potassium phos-

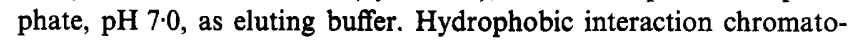
graphy was performed on a column $(100 \times 4.6 \mathrm{~mm})$ of Hydropore HIC 83 (Rainin). Different gradients of n-propyl alcohol in $0 \cdot 1 \mathrm{M}$ triethylammonium acetate buffer, $\mathrm{pH} 7 \cdot 0$, were employed. Flow rates varied from $0 \cdot 1$ to $1 \mathrm{ml} \mathrm{min}^{-1}$, and $200 \mu 1$ fractions were collected. The carbohydrate content of eluates was assayed with the phenol $/ \mathrm{H}_{2} \mathrm{SO}_{4}$ reagent (Dubois et al., 1966).

Analytical procedures. The relative amounts of mannose, arabinose and inositol were estimated by GC of the alditol acetate derivatives (Hunter et al., 1986). In order to establish the absolute configuration of mannose and arabinose, trimethylsilyl derivatives of $R-(-)$ - and $S$ (+)-2-butyl glycosides were analysed by GC/MS with appropriate standards (Gerwig et al., 1978).

Deacylation of LAM $(14 \mathrm{mg})$ was conducted with $0.1 \mathrm{M}-\mathrm{NaOH}$ in $\mathrm{H}_{2} \mathrm{O}$ at $37^{\circ} \mathrm{C}$ for $18 \mathrm{~h}$ (Hunter \& Brennan, 1990). After neutralization with $\mathrm{CH}_{3} \mathrm{COOH}$, lipids were extracted with $\mathrm{CHCl}_{3} / \mathrm{CH}_{3} \mathrm{OH}(2: 1, \mathrm{v} / \mathrm{v})$. To remove salts, the aqueous fraction was applied to a column $(1 \times 100 \mathrm{~cm})$ of Bio-Gel P-100 (Bio-Rad) as described by Hunter \& Brennan (1990), to yield $6.3 \mathrm{mg}$ of purified deacylated LAM (dLAM).

For analysis of glycosyl linkage composition, dLAM (6 mg) was per$O$-methylated as mentioned by Daffé et al. (1990). A fraction ( $0.1 \mathrm{mg})$ was further hydrolysed with $2 \mathrm{M}-\mathrm{CF}_{3} \mathrm{COOH}$ at $120^{\circ} \mathrm{C}$ for $2 \mathrm{~h}$, reduced with $\mathrm{NaB}^{2} \mathrm{H}_{4}$, acetylated and subjected to $\mathrm{GC}$ and $\mathrm{GC} / \mathrm{MS}$ analyses (Daffé et al., 1990; Chatterjee et al., 1991). The remaining material $\left(5.9 \mathrm{mg}\right.$ ) was partially hydrolysed with $2 \mathrm{M}-\mathrm{CF}_{3} \mathrm{COOH}$ at $75^{\circ} \mathrm{C}$ for $1 \mathrm{~h}$ and reduced with $\mathrm{NaB}^{2} \mathrm{H}_{4}$. Fragments of oligoglycosyl alditols thus obtained were $O$-ethylated and partially purified on a SEP-PAK (C-18) cartridge (Waters Associates), by eluting with 35 and $45 \%(\mathrm{v} / \mathrm{v})$ $\mathrm{CH}_{3} \mathrm{CN}$ in $\mathrm{H}_{2} \mathrm{O}$ (Daffé et al., 1990; Chatterjee et al., 1991). Further separation was achieved by reverse-phase HPLC using a column $(4 \times 250 \mathrm{~mm}$ ) of Lichrosorb RP18 (Merck). Fractions of $0.5 \mathrm{ml}$ were collected and either analysed by GC/MS or completely hydrolysed, reduced with $\mathrm{NaB}^{2} \mathrm{H}_{4}$, acetylated and the products identified by GC/MS (Daffé et al., 1990; Chatterjee et al., 1991).

${ }^{13} \mathrm{C}-\mathrm{NMR}$ was conducted in ${ }^{2} \mathrm{H}_{2} \mathrm{O}$ on a Brucker 300 instrument at Colorado State University, Department of Chemistry Central Instrument Facility (Chatterjee et al., 1992b).

Electrophoresis and Western blotting. LAM preparations were routinely examined by analytical SDS-PAGE (Laemmli, 1970). Electrophoresis was conducted on $15 \%(\mathrm{w} / \mathrm{v})$ acrylamide $/ 0.8 \%$ bisacrylamide gels at $10 \mathrm{~mA}$ for $15 \mathrm{~min}$ and $15 \mathrm{~mA}$ for $45 \mathrm{~min}$. Gels were silver stained (Tsai \& Frasch, 1982) with a periodate oxidation step included. Two-dimensional electrophoresis was also performed on purified LAM ( 2 to $4 \mu \mathrm{g}$ ) (O'Farrel, 1975). The first dimension involved isoelectric focusing in $15 \mathrm{M}$-urea with a $8 \%(\mathrm{v} / \mathrm{v})$ ampholyte mixture (Bio-Lyte, Bio-Rad). The second dimension involved SDS-PAGE on a $15 \%$ polyacrylamide gel.

Immunoblotting was conducted on samples of the product from $M$. bovis BCG in parallel with purified LAM $(1,2,4,8 \mu \mathrm{g})$ from $M$. tuberculosis $\mathrm{H} 37 \mathrm{Ra}$ and $M$. tuberculosis Erdman. After electrophoresis, gels were transblotted to nitrocellulose (Bio-Rad) in Tris/glycine/ methanol buffer under $40 \mathrm{~V}$ constant voltage (Gaylord et al., 1987). The nitrocellulose sheets were blocked in Tris-buffered saline (TBS) containing $0.01 \%$ Tween-20 (TBST) and $5 \%(\mathrm{w} / \mathrm{v})$ nonfat dry milk. Several antibodies diluted in TBST were used : an ascitic IgG anti-LAM monoclonal antibody CS-35 (ML9D3) (diluted 1:2000) (Chatterjee et $a l ., 1992 b$ ), a monoclonal antibody ML906 directed to LAM from $M$. leprae (diluted 1:250) (Gaylord et al., 1987), and CS-40 raised to LAM from $M$. tuberculosis Erdman (undiluted) (Chatterjee et al., 1992b). A pool of sera from tuberculous patients (diluted 1:1000, v/v), a serum sample from a nontuberculous patient (diluted $1: 300, \mathrm{v} / \mathrm{v}$ ), three sera from individual tuberculous patients (diluted 1:3000, v/v), three specimens from individual lepromatous leprosy patients (diluted $1: 3000, v / v)$, four bovine serum samples from $M$. bovis-infected animals (diluted $1: 1000, \mathrm{v} / \mathrm{v}$ ), and one bovine serum sample from a non-infected animal (diluted $1: 1000, v / v$ ), were also tested. Antibodies were incubated overnight at room temperature. The nitrocellulose sheets were then washed with TBST and the appropriate antiserum conjugated with alkaline phosphatase (Sigma) was then applied. After a further $1 \mathrm{~h}$ of incubation at room temperature, bound antibodies were detected with 5-bromo-4-chloro-3-indolyl phosphate (BCIP) $/ p$ nitro blue tetrazolium chloride (NBT) substrate (Sigma). Biotinylated concanavalin A (Boehringer Mannheim) was also used at a dilution of $1: 3000(\mathrm{v} / \mathrm{v})$ in phosphate-buffered saline (PBS) containing $0.05 \%$ (v/v) Tween-20 (PBST). Bound concanavalin A was detected with horseradish peroxidase-conjugated streptavidin (Zymed Laboratories) (Gaylord et al., 1987).

\section{Results}

\section{Purification of LAM from $M$. bovis BCG}

After each purification step, the degree of purity of the LAM from $M$. bovis BCG was examined by SDS-PAGE, in which it appeared as a broad, diffuse band. HPLC or 
Table 1. A comparison of the glycosyl composition of LAM from $M$. tuberculosis H37Ra, M. bovis BCG and M. tuberculosis Erdman

\begin{tabular}{|c|c|c|c|c|}
\hline \multirow[b]{2}{*}{ Product identified } & \multirow{2}{*}{$\begin{array}{l}\text { Deduced } \\
\text { form }\end{array}$} & \multicolumn{3}{|c|}{ Mol \% } \\
\hline & & H37Ra* & BCG & Erdman $\dagger$ \\
\hline 1,4-di- $O$-Ac-2,3,5-tri- $O$-Me-arabinitol & t-Araf & 7 & 3 & 2 \\
\hline 1,2,4-tri- $O$-Ac-3,5-di- $O$-Me-arabinitol & 2-Araf & 9 & 11 & $\overline{7}$ \\
\hline 1,4,5-tri- $O$-Ac-2,3-di- $O$-Me-arabinitol & 5-Araf & 43 & 37 & 41 \\
\hline 1,3,4,5-tetra- $O$-Ac-3-O-Me-arabinitol & 3,5-Araf & 12 & 14 & 19 \\
\hline 1,5-di- $O$-Ac-2,3,4,6-tetra- $O$-Me-mannitol & t-Manp & 11 & 19 & 19 \\
\hline 1,5,6-tri-O-Ac-2,3,4-tri-O-Me-mannitol & 6-Manp & 6 & 2 & 4 \\
\hline 1,2,5,6-tetra-O-Ac-3,4,-di-O-Me-mannitol & 2,6-Manp & 9 & 5 & 9 \\
\hline 1,2,5-tri-O-Ac-3,4,6-tri- $O$-Me-mannitol & 2-Manp & $0 \cdot 6$ & 9 & 8 \\
\hline
\end{tabular}

two-dimensional electrophoresis were unable to further resolve the product, and it seems likely that LAM is not a single defined molecule, a view reinforced by evidence of multi-non-reducing termini (see below).

GC/MS of the fatty acyl methyl esters derived after alkali treatment of LAM showed the presence of methylhexadecanoate ( $42 \%$ of fatty acids), methyloctadecanoate $(17 \%)$, and methyltuberculostearate $(41 \%)$ only. GC analysis of the polyol acetates or the alditol acetates from the purified product gave relative amounts of arabinose, mannose and inositol of about 67:32:1, respectively. The absolute configuration of arabinose and mannose was determined by GC/MS analysis of the trimethylsilyl derivatives of $R-(-)$ - and $S-(+)-2$-butyl glycosides and by comparison with appropriate standards, which established that both arabinose and mannose were in the D-configuration.

\section{Glycosyl linkage composition}

In order to determine the linkage and the ring form of the arabinosyl and mannosyl residues, the dLAM was per- $O$-methylated, hydrolysed, reduced, acetylated and analysed by GC and GC/MS (Table 1). The product, 1,4,5-tri- $O$-Ac-2,3-di- $O$-Me-arabinitol, was attributed to 5-linked arabinofuranose units rather than 4-linked arabinopyranose based on the following evidence. When per- $O$-methylated dLAM was partially hydrolysed, reduced, $O$-ethylated and then completely hydrolysed, reduced and acetylated, 4-linked arabinopyranose would have given either 1,5-di-O-Ac-2,3-di-O-Me-4-O- $\mathrm{C}_{2}\left({ }^{2} \mathrm{H}\right)_{5^{-}}$ arabinitol or 4-O-Ac-2,3-di-O-Me-1,5-di-O-C ${ }_{2}\left({ }^{2} \mathrm{H}\right)_{5}$-arabinitol, whereas a 5-linked arabinofuranose would have yielded either 1,4-di-O-Ac-2,3-di-O-Me-5-O- $\mathrm{C}_{2}\left({ }^{2} \mathrm{H}\right)_{5}$ arabinitol or 5-O-Ac-2,3-di-O-Me-1,4-di- $O-\mathrm{C}_{2}\left({ }^{2} \mathrm{H}\right)_{5}$-arabinitol. Only the two latter compounds were found after hydrolysis of fragments $4,11,13,16,22$ (Table 2). Likewise, 1,3,4,5-tetra- $O$-Ac-2-O-Me-arabinitol proved to be indicative of 3,5-linked arabinofuranose rather than 3,4-linked arabinopyranose since the actual product identified upon hydrolysis of fragments 11 and 27 was 5$O$-Ac-2-O-Me-1,3,4-tri- $O-\mathrm{C}_{2}\left({ }^{2} \mathrm{H}\right)_{5}$-arabinitol; 3,4-linked arabinopyranose would have given rise to 4-O-Ac-2-O$\mathrm{Me}-1,3,5$-tri- $\mathrm{O}-\mathrm{C}_{2}\left({ }^{2} \mathrm{H}\right)_{5}$-arabinitol, which was never found in the hydrolysate from any of the fragments. Thus all of the arabinosyl residues are apparently furanoid and 2-, 5- or 3,5-linked. There were also some small amounts (approx. 3\%) of terminal arabinofuranose. The mannosyl residues were clearly in the pyranose form, 2-, 6- or 2,6-linked. Terminal nonreducing mannopyranose was also present and accounted for about $19 \%$ of total sugars.

A comparison of the glycosyl composition of LAM from $M$. tuberculosis H37Ra, M. tuberculosis Erdman and $M$. bovis BCG is shown in Table 1. LAM from BCG and $M$. tuberculosis Erdman shared a similar sugar profile. However, the percentages of each form were noticeably different. The 6- and 2,6-linked mannopyranosyl constituents reflective of the mannan backbone (Chatterjee et al., 1991) represented only $7 \%$ of the total sugar content of LAM from BCG, instead of the $15 \%$ and the $13 \%$ for LAM from H37Ra and Erdman, respectively. Moreover, the large amount of 3,5-linked arabinofuranose in the BCG product and the presence of only a few terminal arabinofuranose residues supported the existence of mannose-capped arabinosyl side chains which themselves must be highly branched. In summary, the data in Table 1 indicate that the mannan backbone of LAM from BCG is short, the arabinofuranosyl side chains are highly branched and mannosyl residues dominate the non-reducing ends of these side chains.

The regions of the anomeric carbons in the ${ }^{13} \mathrm{C}-\mathrm{NMR}$ spectrum of LAM from $M$. bovis BCG are shown in Fig. 1 . The chemical shifts at $\delta 108 \cdot 7, \delta 108 \cdot 3$ and $\delta 106 \cdot 7$ are ascribed to C-1 of 5-, 3,5- and 2-linked $\alpha$-arabinofuranose units, respectively (Daffé et al., 1990; Chatterjee et al., 
Table 2. Proposed structures for the fragments arising from ManLAM of $M$. bovis $B C G$

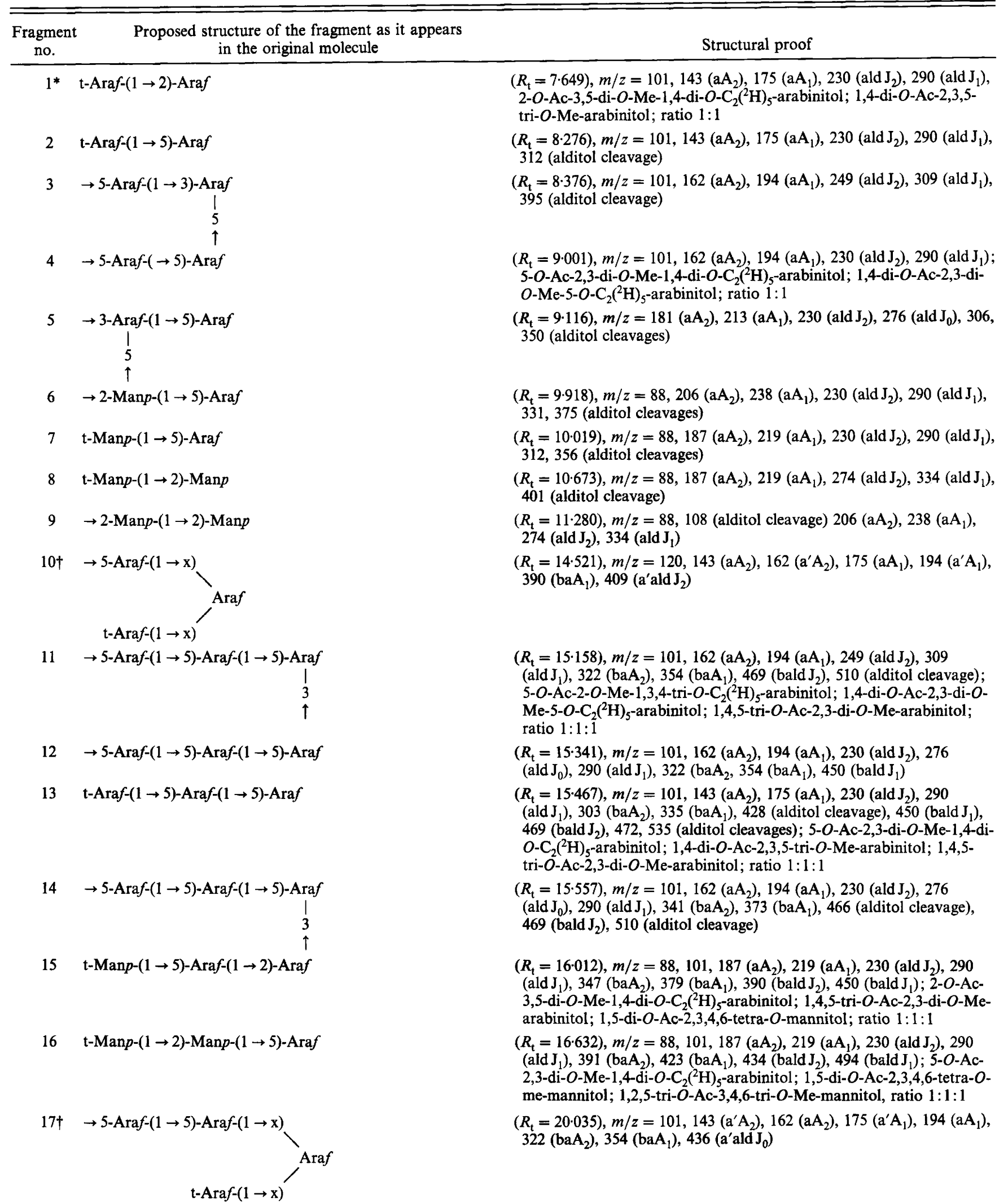


Table 2 (cont.)

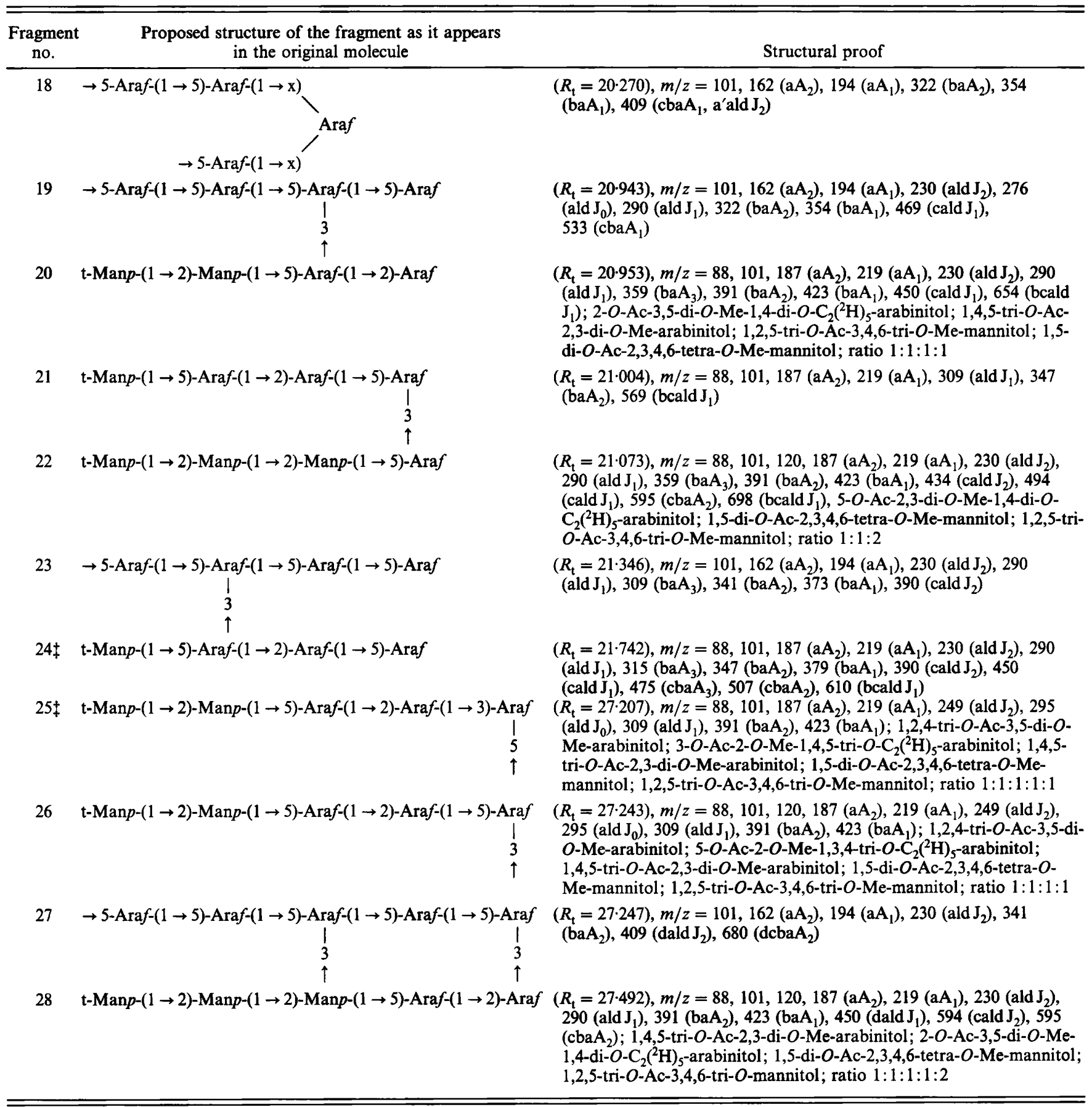

* Partially $O$-methylated, partially $O$-pentadeuterioethylated glycosyl alditols were separated by HPLC, and further resolved and analysed by GC/MS. Also, in order to determine the glycosyl linkage composition, each fragment was hydrolysed, reduced, acetylated and submitted to GC/MS. Thus for example, fragment 5 was identified as $\left.2-O-\mathrm{C}_{2}{ }^{2} \mathrm{H}\right)_{5}-3,4,6$-tri-O-Me-Manp-(1 $\left.\rightarrow 2\right)-1,5-\mathrm{di}-\mathrm{O}-\mathrm{C}_{2}\left({ }^{2} \mathrm{H}\right)_{5}-3,4,6-$ tri-O-Me-mannitol, which points to the presence of $\rightarrow 2$-Manp- $(1 \rightarrow 2)$-Man $p$ in the original product.

$\dagger \mathrm{x}$ is probably 3 and 5 . However, we could not categorically differentiate between the two. 20 .

$\ddagger$ The linkage between the Araf residues was not independently established, but was based on knowledge of the structures of compounds 15 and

$1992 b$ ), whereas signals around $\delta 101 \cdot 7$ are due to $C-1$ of terminal and 5-linked $\beta$-arabinofuranose units (Chatterjee $e t$ al., 1992b). On the basis of results from Lipkind $e t$ al. (1988), chemical shifts of carbon from polysaccharide sources may be calculated. Thus, the signal at $\delta 103.5$ was ascribed to the anomeric carbon of $t-\alpha$-mannopyra- 


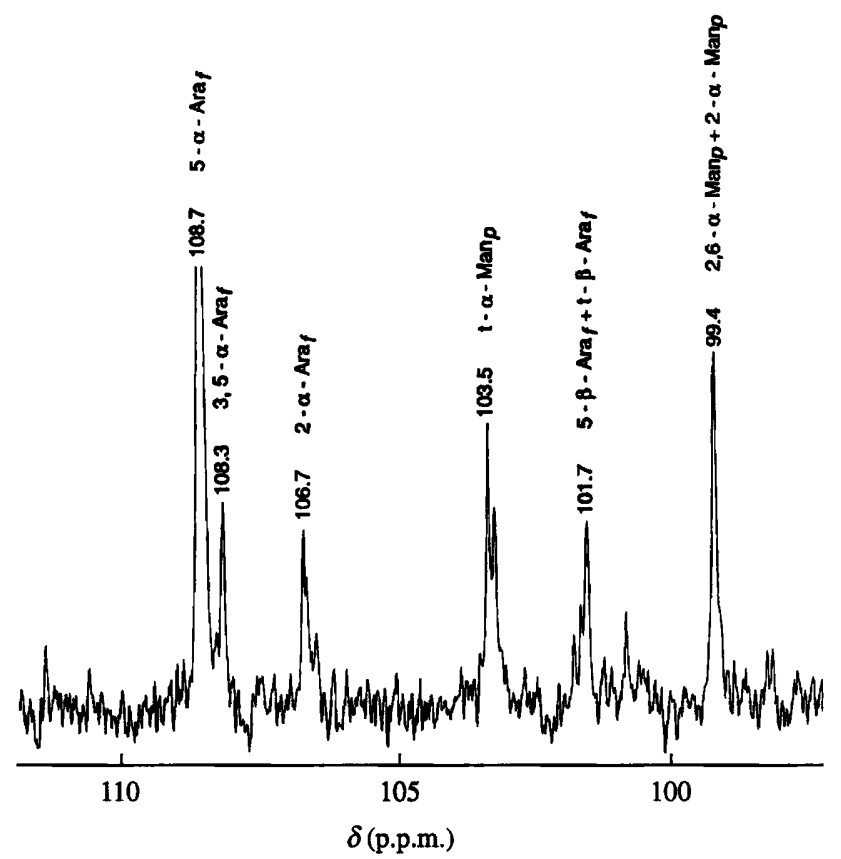

Fig. $1 .{ }^{13} \mathrm{C}$-NMR of LAM from $M$. bovis BCG showing the anomeric regions only (between 95 and 110 p.p.m.).

nose from $\mathrm{t}-\alpha-\mathrm{D}-\mathrm{Man} p-(1 \rightarrow 2)-\alpha-\mathrm{D}-\mathrm{Man} p$ (calculated value $\delta 103.4)$ and $\delta 99.4$ to $\mathrm{C}-1$ of 2,6 -linked- $\alpha$-Dmannopyranose from 2,6- $\alpha$-D-Man $p-(1 \rightarrow 6)-\alpha-\mathrm{D}-\operatorname{Man} p$ (calculated value $\delta 99.4)$. The anomeric carbon of 2linked mannopyranose can also resonate at $\delta 99.4$ (Chatterjee et al., 1992b). The ${ }^{13} \mathrm{C}-\mathrm{NMR}$ spectra corroborate the results deduced from the methylation analysis, namely that all arabinosyl residues are in the furanosyl form and that the mannosyl residues are in the pyranosyl ring form.

\section{Evidence for the presence of terminal mannosyl residues} in $L A M$ from $M$. bovis $B C G$

Per- $O$-methylated dLAM was partially hydrolysed. The fragments obtained were reduced, $O$-ethylated and isolated by reverse-phase HPLC. They were either further analysed by GC/MS or completely hydrolysed, reduced, acetylated and identified by GC/MS (Daffé et al., 1990). With such a protocol, only one-third of the furanosyl residues were hydrolysed; the mannan backbone remained intact (Chatterjee et al., 1991). A total of 28 fragments were characterized (Table 2); the evidence leading to structural elucidation is provided for fragments 20 and 28 in Figs 2 and 3. GC/MS showed that fragment 20 (Fig. 2) consisted of tetra- $O$-Me-Man $\rightarrow$ tri$O$-Me-Man $\rightarrow$ di-O-Me-Ara $\rightarrow$ di- $O$-Me-1,5-di- $O-\mathrm{C}_{2}\left({ }^{2} \mathrm{H}\right)_{5^{-}}$ arabinitol. Hydrolysis, reduction and acetylation gave rise to 2-O-Ac-3,5-di-O-Me-1,4-di- $O-\mathrm{C}_{2}\left({ }^{2} \mathrm{H}\right)_{5}$-arabinitol, 1,4,5-tri-O-Ac-2,3-di- $O$-Me-arabinitol, $\quad$ 1,2,5-tri-O-Ac-

(a)

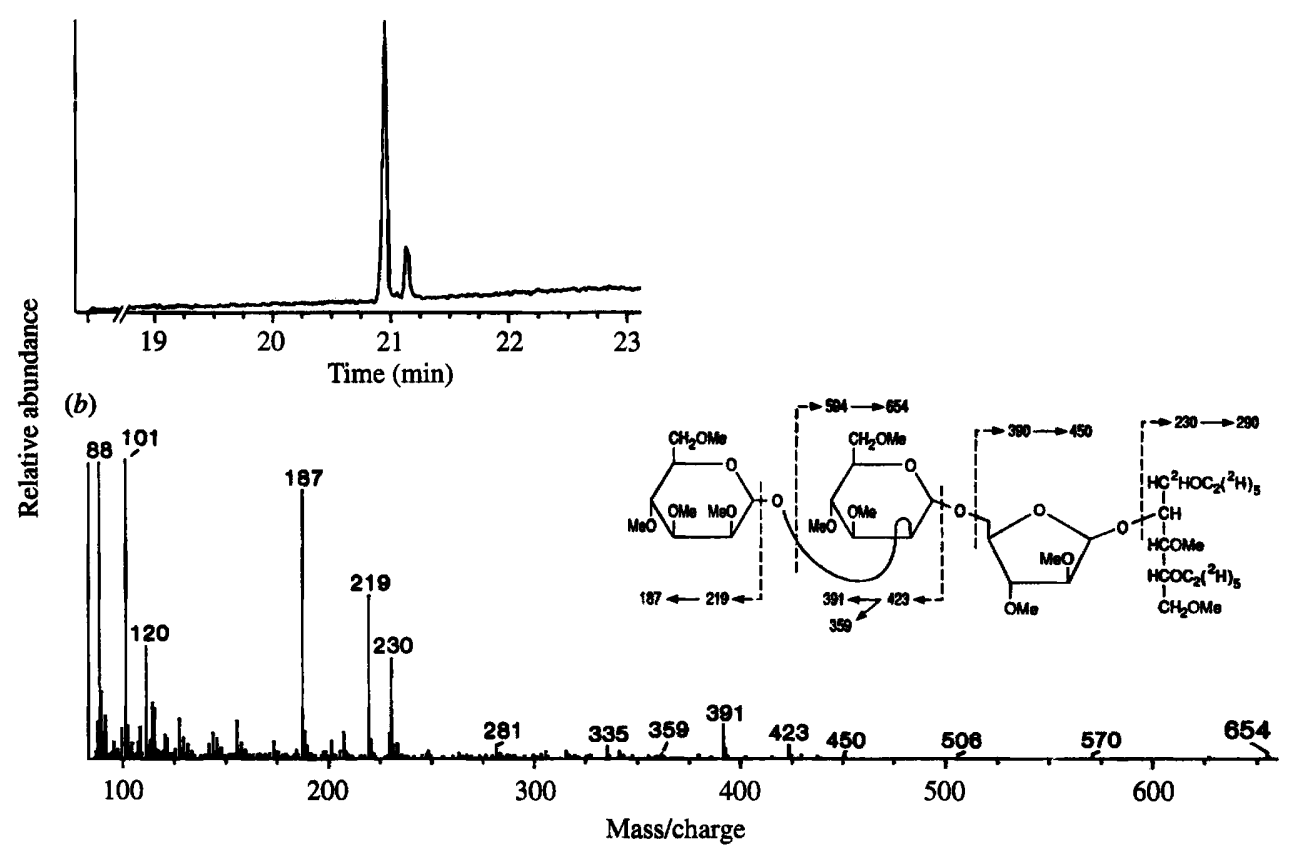

Fig. 2. Analysis of compound 20. (a) Total ion chromatography. (b) Mass spectrum and structural interpretation of the partially $O$ -

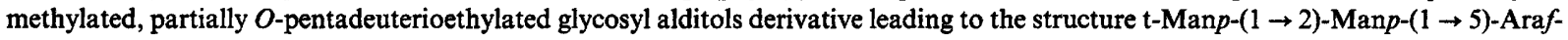
$(1 \rightarrow 2)$-Araf. 
(a)

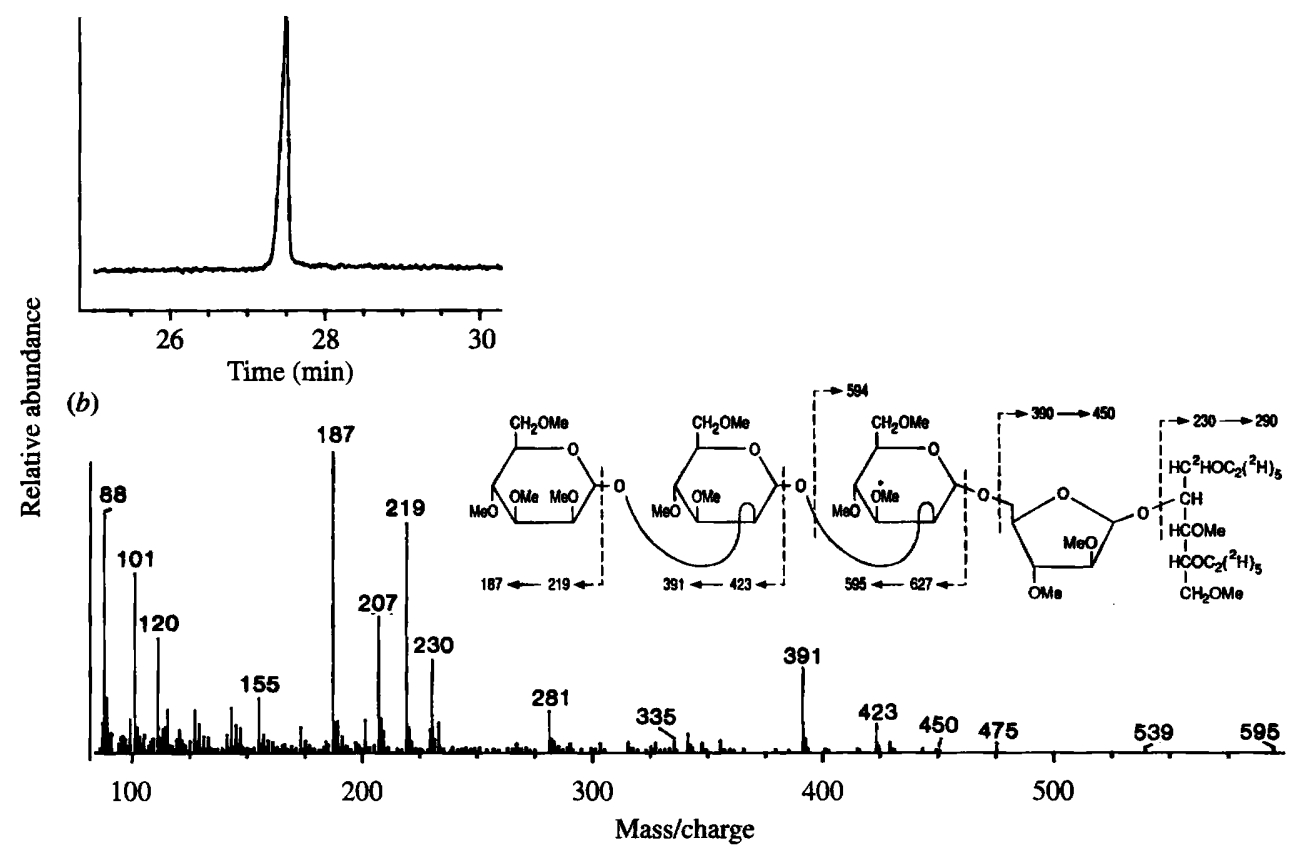

Fig. 3. Analysis of compound 28. (a) Total ion chromatography. (b) Mass spectrum and structural interpretation of the partially $O$ methylated, partially $O$-pentadeuterioethylated glycosyl alditols derivative leading to the structure t-Man $p$ - $(1 \rightarrow 2)-\operatorname{Man} p-(1 \rightarrow 2)-$ Manp- $(1 \rightarrow 5)$-Araf- $(1 \rightarrow 2)$-Araf.

3,4,6-tri-O-Me-mannitol and 1,5-di-O-Ac-2,3,4,6-tetra$O$-Me-mannitol in the ratio of approximately $1: 1: 1: 1$. Thus, compound 20 is $2,3,4,6$-tetra-O-Me-Man $p-(1 \rightarrow$ 2)-3,4,6-tri- $O$-Me-Man $p$-( $1 \rightarrow 5$ )-2,3-di- $O$-Me-Araf-( $1 \rightarrow$ 2)-3,5-di-O-Me-1,4-di- $O-\mathrm{C}_{2}\left({ }^{2} \mathrm{H}\right)_{5}$-arabinitol, pointing to the presence of the fragment $\mathrm{t}-\mathrm{Man} p-(1 \rightarrow 2)-\operatorname{Man} p-(1 \rightarrow$ $5)$-Araf- $(1 \rightarrow 2)$-Araf in dLAM. Likewise the hydrolysis of fragment 28 (Fig. 3) produced 1,4,5-tri-O-Ac-2,3-di$O$-Me-arabinitol, 2-O-Ac-3,5-di-O-Me-1,4-di-O-C ${ }_{2}\left({ }^{2} \mathrm{H}\right)_{5}$ arabinitol, 1,5-di-O-Ac-2,3,4,6-tetra-O-Me-mannitol and 1,2,5-tri- $O$-Ac-3,4,6-tri- $O$-Me-mannitol in the approximate ratio of $1: 1: 1: 2$. Thus the complete structure of fragment 28 is 2,3,4,6-tetra-O-Me-Manp-( $1 \rightarrow 2)$ 3,4,6-tri- $O$-Me-Man $p$ - $(1 \rightarrow 2)-3,4,6$-tri- $O$-Me-Man $p$ $(1 \rightarrow 5)$-2,3-di- $O$-Me-Araf-( $\rightarrow$ 2)-3,5-di- $O$-Me-1,4-di- $O$ $\mathrm{C}_{2}\left({ }^{2} \mathrm{H}\right)_{5}$-arabinitol, pointing to the presence of the unit t$\operatorname{Man} p-(1 \rightarrow 2)-M a n p-(1 \rightarrow 2)-M a n p-(1 \rightarrow 5)$-Araf- $(1 \rightarrow$ 2)-Araf in LAM from $M$. bovis BCG.

Of the 28 fragments, 11 contained terminal mannoses, again emphasizing the extent of mannose capping of the putative terminal arabinofuranosyl units in this product. Thus, from fragments 7,15,21 and 24, it was evident that stretches of Araf are substituted with single mannose or dimannosyl units (evident from fragments 20,25 and 26) or trimannosyl units (22 and 28). Mannosyl residues were all linked together by a $1 \rightarrow 2$ linkage $(6,8,9,16,20$, $22,25,26,27)$. In agreement with the results obtained with LAM from $M$. tuberculosis Erdman, the mannosyl side chain was often linked to an arabinose within the Ara- $(1 \rightarrow 5)$-Ara- $(1 \rightarrow 2)$-Ara motif $(15,20,21,24,25,26$, 28). The arabinan side chain showed the frequent occurrence of the diarabinosyl unit, Ara-( $\rightarrow 5)$-Ara, and 13 fragments in all contained 3,5-linked arabinose $(3,5,10,11,14,17,18,19,21,23,25,26,28)$. The structural features of several of the tetrasaccharides and pentasaccharides demonstrated that the mannosyl residues were invariably bound to just two or three linear arabinofuranosyl units prior to the 3,5-linked branched arabinofuranose unit.

\section{Western blotting (immunoblotting) of LAM from $M$.} bovis $B C G$

Immunoblotting (Fig. 4), demonstrated that concanavalin A bound to LAM from BCG and $M$. tuberculosis Erdman with equal intensity but not to LAM of H37Ra, providing further evidence that LAM from BCG did contain mannosyl residues at the non-reducing end of the molecule. The antigenicity of LAM from BCG was examined by immunoblotting and by comparison with LAM from $M$. tuberculosis Erdman and $M$. tuberculosis H37Ra (Fig. 4). All products reacted strongly against pooled sera from tuberculosis patients, and against serum specimens from individual tuberculosis patients, lepromatous patients and $M$. bovis-infected animals. Thus the inherent antigenicity of ManLAM from $M$. 


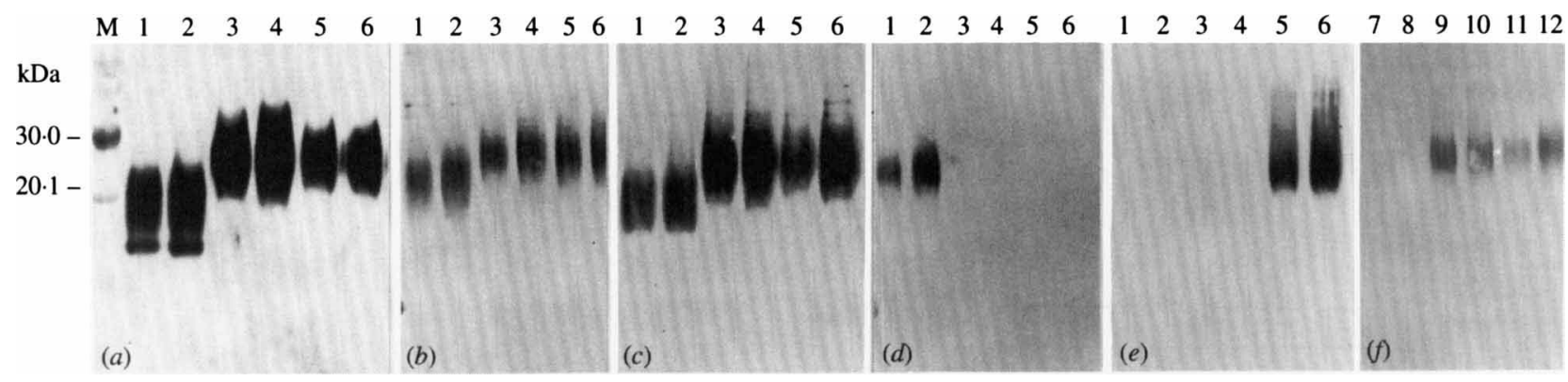

Fig. 4. SDS-PAGE and Western blot of LAM from M. tuberculosis H37Ra, M. bovis BCG and M. tuberculosis Erdman. Lanes: 1 (1 $\mu \mathrm{g})$, $2(2 \mu \mathrm{g}), 7(4 \mu \mathrm{g}), 8(8 \mu \mathrm{g})$ of $M$. tuberculosis H37Ra LAM; $3(1 \mu \mathrm{g}), 4(2 \mu \mathrm{g}), 9(4 \mu \mathrm{g}), 10(8 \mu \mathrm{g})$ of $M$. bovis BCG LAM; $5(1 \mu \mathrm{g}), 6$ $(2 \mu \mathrm{g}), 11(4 \mu \mathrm{g}), 12(8 \mu \mathrm{g})$ of $M$. tuberculosis Erdman LAM. (a) Gel stained with silver nitrate. (b-f) Immunoblotting using $(b)$ pooled tuberculous patients' sera (diluted 1:1000), (c) monoclonal antibody CS-35 (diluted 1:2000), (d) monoclonal antibody ML906 (1:250), (e) monoclonal antibody CS-40 (undiluted), $(f)$ concanavalin A (diluted 1:3000). The positions of polypeptide markers (soybean trypsin inhibitor: $20 \cdot 1 \mathrm{kDa}$, carbonic anhydrase: $30 \mathrm{kDa}$ ) are indicated in lane $\mathrm{M}$.

bovis was demonstrated and so was its extensive crossreactivity. Nevertheless, the use of monoclonal antibodies demonstrated the existence of elements of antigenic specificity in the various products. LAM from the three different sources reacted strongly against a monoclonal antibody (CS-35) originally raised to LAM from $M$. leprae. However, only the LAM from $M$. tuberculosis $\mathrm{H} 37 \mathrm{Ra}$ reacted with another anti-M. leprae LAM antibody, ML906. In this case neither the product from Erdman nor that from BCG was recognized even when large amounts $(8 \mu \mathrm{g})$ of LAM were loaded. The antibody CS-40, which had been raised against LAM from Erdman, surprisingly did not cross-react with the product from either $\mathrm{H} 37 \mathrm{Ra}$ or $\mathrm{BCG}$, even when large amounts $(10 \mu \mathrm{g})$ were tested.

\section{Discussion}

To date, two major classes of lipoarabinomannan from various mycobacterial sources have been described: LAM (AraLAM) which possesses arabinofuranosecontaining termini in either of two arrangements, $\beta$-DAraf- $(1 \rightarrow 2)-\alpha$-D-Araf-( $1 \rightarrow 5)-\alpha$-D-Araf or [ $\beta$-D-Araf$(1 \rightarrow 2)-\alpha-D-A r a f-(1 \rightarrow]_{2}-(3$ and 5$)-\alpha$-D-Araf, and which was found in an avirulent strain subcultured in laboratory media for many years and thought to be $M$. tuberculosis H37Ra (Chatterjee et al., 1991); and ManLAM, in which the arabinan ends are extensively capped with single mannosyl, di-, tri- or tetramannosyl units and which was observed in virulent $M$. tuberculosis Erdman (Chatterjee et al., 1992b). In the light of this dramatic difference between LAM from the two strains, we had speculated that the mannose adducts were of relevance to pathogenicity.

However, in view of the present evidence that LAM from $M$. bovis BCG is also of the ManLAM class, a direct relationship between mannose capping and patho- genesis is hardly tenable. Indeed, arising directly from this study, we now question the authenticity of our $M$. tuberculosis $\mathrm{H} 37 \mathrm{Ra}$ strain used in the initial work (Chatterjee et al., 1991). It was always much more rapidly growing than other strains of $M$. tuberculosis H37Ra (Chatterjee et al., 1992a,b). We have since shown that it is devoid of the majority of the key biochemical characteristics of H37Ra. In particular, the pattern of mycolic acids as their methyl esters on thin layer plates, conducted as described by Minnikin et al. (1975), showed the complete absence of methoxy mycolates, a hallmark of strains of $M$. tuberculosis and $M$. bovis. Thus, we conclude that the strain in the earlier study was a rapidly growing Mycobacterium $\mathrm{sp}$. and that the absence of mannose capping is probably more a feature of rapid growth than of pathogenesis.

The relationship between structure and biological properties of LAM has been discussed. Several structural features are thought to play a key role in biological activity, such as the $\alpha-(1 \rightarrow 5)$-linked arabinosyl moieties, the $O$-acyl groups and the mannose capping. For example, it is known that $\alpha-(1 \rightarrow 5)$-linked arabinosyl residues located on the arabinan side chains are the main immunodeterminants, since the deacylated form of LAM, i.e. arabinomannan (and also arabinogalactan), after treatment with arabinases lost their immunological activity (Misaki et al., 1977). Also, lipomannan has neither immunological activity (Gaylord et al., 1987) nor capacity to induce secretion of TNF- $\alpha$ (Moreno et al., 1989). Moreover, Moreno et al. (1988) found that LAM, as well as arabinomannan and D-arabino-D-galactan, but not L-arabino-L-galactan, were able to inhibit T-cell proliferation of human clones, leading to the hypothesis that the inhibition of major cellular immune functions by LAM is due to the polysaccharide region rather than the $O$-acyl groups. More recently (Chatterjee $e t$ al., 1992c), it has been found that AraLAM was 100-fold more 


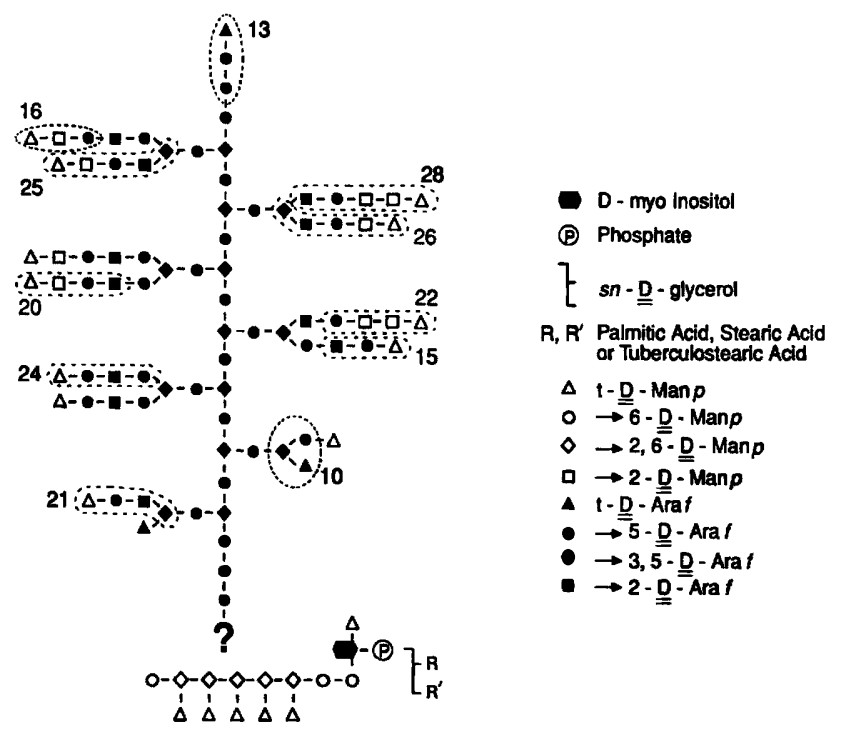

Fig. 5. A hypothetical model representing the major structural features of LAM from $M$. bovis BCG.

effective in inducing TNF secretion than ManLAM, supporting the view that the masking of terminal arabinosyl residues by mannose directly influences biological activity. In addition, the $O$-acyl groups of LAM are thought to be involved in its biological properties. For example, de- $O$-acylation with alkali diminishes the release of TNF in vitro (Moreno et al., 1989) and inhibits $\gamma$-interferon-mediated activation of mouse macrophages in vivo (Sibley et al., 1988).

The results presented in the present paper demonstrate that the non-reducing termini of LAM from $M$. bovis BCG are mannose capped. Moreover, the data presented in Table 1 are consistent with the following structural features in BCG LAM: (i) a short mannan backbone with only 5 units of 2,6-linked mannose; (ii) an arabinofuranosyl side chain highly branched due to a plenitude of 3,5-linked D-Araf residues; and (iii) up to three 2-linked mannopyranosyl segments located at the non-reducing ends of the molecule. A model which accounts for most of the observed features of LAM from BCG is presented in Fig. 5.

Among fragments obtained after per- $O$-methylation and partial hydrolysis of BCG LAM, many correspond to motifs previously described for LAM from H37Ra (Chatterjee et al., 1991) or from Erdman (Chatterjee $e t$ $a l ., 1992 b$ ). Although motifs containing terminal arabinofuranose (motif A) or long linear chains of arabinofuranose (motifs $C$ and D) are not major components of LAM from BCG, fragments containing 3,5-linked Araf (motif B) are well represented. More outstanding are the similarities of motifs E, F, G from Erdman LAM with fragments 15, 20 and 28 from BCG LAM. However, there is no evidence for the presence of a tetramannosyl- containing domain (motif $\mathrm{H}$ ) in LAM from BCG. Thus, like that from H37Ra, LAM from BCG seems to be highly branched but, like that from Erdman, it possesses mannosyl domains at the non-reducing end of the molecule.

The antigenicity of LAM from BCG, and especially its reactivity with tuberculous patient sera and with some monoclonal antibodies, underlines again the well-known cross-reactivity of LAM. On the other hand, unlike LAM from H37Ra, that from BCG and M. tuberculosis Erdman did not react with yet another anti- $M$. leprae LAM antibody (ML906). The structural similarities between LAM from BCG and Erdman, more particularly the presence in both molecules of mannosyl domains on the arabinosyl side chain, may account for their similar antigenic behaviour. It was thus expected that an antibody raised against LAM from Erdman would recognize LAM from BCG. Unexpectedly, no reaction was observed between an anti-Erdman LAM antibody (CS-40) and BCG LAM, even at high concentration. In addition mouse serum raised against sonicated $M$. avium reacted with $M$. avium ManLAM but not with ManLAM from $M$. tuberculosis Erdman or $M$. bovis BCG (unpublished results). Thus, although LAM from BCG shows considerable structural analogies with Erdman LAM, it also shows antigenic differences, which are indicative of yet other niceties, such as: (i) some saccharide motifs (e.g. motif $\mathrm{H}$ ) may exist only in Erdman LAM and not in BCG or H37Ra LAM; (ii) differences may lie in the involvement of acyl, acetyl or phosphoryl functions. It is noteworthy that the dominant structural features of LAM were established after deacylation and since deacylated LAM does not react with any antibodies, the nature of such functions and their roles in epitope recognition were not clearly investigated. The evidence for antigenic specificity within the ManLAMs from different mycobacteria further underlines the need for further immunochemical investigation of these molecules.

We would like to thank Becky Rivoire and Kristin Lowell for technical help, Michael McNeil for helpful discussions, Carol Marander and Greg Nelson for graphics, and Marilyn Hein for preparation of the manuscript. The work was funded by grants AI-18357 and AI-27288, and contract AI-05074 from the National Institute of Allergy and Infectious Diseases, National Institutes of Health.

\section{References}

Barnes, P. F., Fong, S.-J., Brennan, P. J., Twomey, P. E., MazumDER, A. \& MODLIN, R. L. (1990). Local production of tumor necrosis factor and INF- $\gamma$ in tuberculous pleuritis. Journal of Immunology 145, 149-154.

Barnes, P. F., Chatterjee, D., Brennan, P. J., Rea, T. H. \& Modlin, R. L. (1992). Tumor necrosis factor production in patients with leprosy. Infection and Immunity 60, 1441-1446.

Chan, J., Fan, X., Hunter, S. W., Brennan, P. J. \& Bloom, B. R. 
(1991). Lipoarabinomannan, a possible virulence factor involved in persistence of Mycobacterium tuberculosis within macrophages. Infection and Immunity 59, 1755-1761.

Chatterjee, D., Bozic, C. M., McNeil, M. \& Brennan, P. J. (1991). Structural features of the arabinan component of the lipoarabinomannan of Mycobacterium tuberculosis. Journal of Biological Chemistry 266, 9652-9660.

Chatterjee, D., Hunter, S. W., McNeil, M. \& Brennan, P. J. (1992a). Lipoarabinomannan. Multiglycosylated form of the mycobacterial mannosylphosphatidylinositols. Journal of Biological Chemistry 267, 6228-6233.

Chatterjee, D., Lowell, K., Rivoire, B., McNeil, M. \& Brennan, P. J. (1992b). Lipoarabinomannan of Mycobacterium tuberculosis. Capping with mannosyl residues in some strains. Journal of Biological Chemistry 267, 6234-6239.

ChatterJee, D., Roberts, A. D., Lowell, K., Brennan, P. J. \& ORMe, I. (1992c). Structural basis of lipoarabinomannan to induce secretion of tumor necrosis factor. Infection and Immunity 60, 1249-1253.

Chujor, C. S. N., KuHN, B., SchWerer, B., Bernheimer, H., LeVIS, W. R. \& BEVEC, D. (1992). Specific inhibition of mRNA accumulation for lymphokines in human $T$ cell line Jurkat by mycobacterial lipoarabinomannan antigen. Clinical and Experimental Immunology 87, 398-403.

DaffÉ, M., Brennan, P. J. \& MCNeill, M. (1990). Predominant structural features of the cell wall arabinogalactan of Mycobacterium tuberculosis as revealed through characterization of oligoglycosyl alditol fragments by gas chromatography/mass spectrometry and by ${ }^{1} \mathrm{H}$ and ${ }^{13} \mathrm{C}$ NMR analyses. Journal of Biological Chemistry 265, 6734-6743.

Dubois, M., Gilles, K. A., Hamilton, J. K., Rebers, P. A. \& Smith, F. (1966). Colorimetric method for determination of sugars and related substances. Analytical Chemistry 28, 350-356.

Gaylord, H., Brennan, P. J., Young, D. B. \& Buchanan, T. M. (1987). Most Mycobacterium leprae carbohydrate-reactive monoclonal antibodies are directed to lipoarabinomannan. Infection and Immunity 55, 2860-2863.

GeRWig, G. J., Kamerling, J. P. \& Vliegenthart, J. F. G. (1978). Determination of the $\mathrm{D}$ and $\mathrm{L}$ natural configuration of monosaccharides by high-resolution capillary g.l.c. Carbohydrate Research 62, 349-357.

HUNTER, S. W. \& BrenNAN, P. J. (1990). Evidence for the presence of a phosphatidylinositol anchor on the lipoarabinomannan and lipomannan of Mycobacterium tuberculosis. Journal of Biological Chemistry 265, 9272-9279.

Hunter, S. W., GaYloRd, H. \& Brennan, P. J. (1986). Structure and antigenicity of the phosphorylated lipopolysaccharide antigens from the leprosy and tubercle bacilli. Journal of Biological Chemistry 261, 12345-12351.

Kaplan, G., Gandhi, R. R., Weinstein, D. E., LeVis, W. R., Patarroyo, M. E., Brennan, P. J. \& CohN, Z. A. (1987). Mycobacterium leprae antigen-induced suppression of $\mathrm{T}$ cell proliferation in vitro. Journal of Immunology 138, 3028-3034.

LAEMMLI, U. K. (1970). Cleavage of structural proteins during the assembly of the head of bacteriophage T4. Nature, London 227, 680-685.

Levis, W. R., Meeker, H. C., Schuller-Levis, G., Sersen, E., Brennan, P. J. \& FrIED, P. (1987). Mycobacterial carbohydrate antigens for serological testing of patients with leprosy. Journal of Infectious Diseases 156, 763-769.

LiPkind, G. M., Shashkov, A. S., Knirel, Y. A., Vinogradov, E. V. \& KoCHETKOV, N. K. (1988). A computer-assisted structural analysis of regular polysaccharides on the basis of ${ }^{13}$ C-N.M.R. data. Carbohydrate Research 175, 59-75.

Minnikin, D. E., Alshamaony, L. \& Goodfellow, M. (1975). Differentiation of Mycobacterium, Nocardia and related taxa by thin layer chromatographic analysis of whole cell methanolysates. Journal of General Microbiology 88, 200-204.

Misaki, A., Azuma, I. \& YamamuRA, Y. (1977). Structural and immunochemical studies on D-arabino-D-mannans and D-mannans of Mycobacterium tuberculosis and other Mycobacterium species. Journal of Biochemistry 82, 1759-1770.

Molloy, A., Gaudernack, G., Levis, W. R., Cohn, Z. A. \& Kaplan, G. (1990). Suppression of T-cell proliferation by Mycobacterium leprae and its products: the role of lipopolysaccharide. Proceedings of the National Academy of Sciences of the United States of America 87, 973-977.

Moreno, C., Mehlert, A. \& Lamb, J. (1988). The inhibitory effects of mycobacterial lipoarabinomannan and polysaccharides upon polyclonal and monoclonal human $\mathrm{T}$ cell proliferation. Clinical and Experimental Immunology 74, 206-210.

Moreno, C., Taverne, J., Mehlert, A., Bate, C. A. W., Brealey, R. J., Meager, A., Rook, G. A. W. \& Playfair, J. H. L. (1989). Lipoarabinomannan from Mycobacterium tuberculosis induces the production of tumor necrosis factor from human and murine macrophages. Clinical and Experimental Immunology 76, 240-245.

O'FARREL, P. H. (1975). High resolution two-dimensional electrophoresis of proteins. Journal of Biological Chemistry 250, 4007-4021.

Sibley, L. D., Hunter, S. W., BrenNan, P. J. \& Krahenbuhl, J, L. (1988). Mycobacterial lipoarabinomannan inhibits gamma interferon-mediated activation of macrophages. Infection and Immunity 56, 1232-1236.

SibleY, L. D., ADAMS, L. B. \& KRAHENBUHL, J. L. (1990). Inhibition of interferon-gamma-mediated activation in mouse macrophages treated with lipoarabinomannan. Clinical and Experimental Immunology 80, 141-148.

Sugden, E. A., Samagh, B. S., Bundle, D. R. \& Duncan, J. R. (1987). Lipoarabinomannan and lipid-free arabinomannan antigens of Mycobacterium paratuberculosis. Infection and Immunity $\mathbf{5 5}$, $762-770$.

Tsai, C. M. \& Frasch, C. M. (1982). A sensitive silver stain for detecting lipopolysaccharides in polyacrylamide gels. Analytical Biochemistry 119, 115-119. 